\title{
Simple parameterization of nuclear attenuation data
}

\author{
N. Akopov, L. Grigoryan, and Z. Akopov \\ Yerevan Physics Institute, Br.Alikhanian 2, 375036 Yerevan, Armenia
}

\begin{abstract}
Based on the nuclear attenuation data obtained by the HERMES experiment on nitrogen and krypton nuclei, it is shown that the nuclear attenuation $R_{M}^{h}$ can be parametrised in a form of a linear polynomial $P_{1}=a_{11}+\tau a_{12}$, where $\tau$ is the formation time, which depends on the energy of the virtual photon $\nu$ and fraction of that energy $z$ carried by the final hadron. Three widely known parameterizations for $\tau$ were used for the performed fit. The fit parameters $a_{11}$ and $a_{12}$ do not depend on $\nu$ and $z$.
\end{abstract}

PACS numbers: 13.87.Fh, 13.60.-r, 14.20.-c, 14.40.-n

Keywords: nuclei, attenuation, quark, formation time

\section{INTRODUCTION}

Semi-inclusive Deep Inelastic Scattering (DIS) of leptons on nuclear targets is widely used for studies of the hadronization process [1, 2, 3, 4]. It is most effective to observe at moderate energies of the virtual photon, when the formation time of the hadron is comparable with the nuclear radius.

Hadronization is the process through which partons, created in an elementary interaction, turn into the hadrons that are observed experimentally. According to theoretical estimates the hadronization process occurs over length scales that vary from less than a femtometer to several tens of femtometers. Hadronization in a nuclear environment is interesting due to two main reasons. First of all, it allows to study the parameters governing this process on the early stage; on the other hand, it can provide initial conditions for the investigation of hadronization in hot nuclear matter, which arises in high energy ion-ion collisions.

The most convenient observable measured experimentally for this process is the Nuclear Attenuation (NA) ratio, which is a ratio of multiplicities on nucleus and deuterium (per nucleon). Next step is to find a variable which allows to present the NA ratio in the most simple form.

We propose the formation time $\tau$ as the best variable for the NA and show that the data can be parametrised in the form of a linear polynomial $P_{1}=a_{11}+\tau a_{12}$. Formation time $\tau$ depends on the energy of photon $\nu$ and fraction of this energy $z=E_{h} / \nu$ carried by the final hadron with energy $E_{h}$. Three widely known parameterizations for $\tau$ were used for the fit procedure. The parameters $a_{11}$ and $a_{12}$, obtained from the fit, do not depend on $\nu$ and $z$. They are functions of the prehadron-nucleon and hadron-nucleon cross sections and the atomic mass number.

The NA data for pions on nitrogen and for identified hadrons on krypton nuclei obtained by the HERMES experiment [3, 4] were used to perform the fit.

This paper is organized as follows. Nuclear attenuation in absorption model is presented in the next section. In Section 3 it is discussed a choice of the appropriate form for the variable $\tau$. Section 4 presents results of the fit. Conclusions are given in Section 5.

\section{NUCLEAR ATTENUATION IN ABSORPTION MODEL}

The semi-inclusive DIS of lepton on nucleus of atomic mass number $\mathrm{A}$ is:

$$
l_{i}+A \rightarrow l_{f}+h+X,
$$

where $l_{i}\left(l_{f}\right)$ are the initial (final) leptons, and $\mathrm{h}$ is the hadron observed in the final state. This process is usually investigated in terms of the NA ratio, which is frequently defined as a function of two variables, $\nu$ and $z^{1}$

$$
R_{M}^{h}(\nu, z) \approx 2 d \sigma_{A}(\nu, z) / A d \sigma_{D}(\nu, z) .
$$

Usually it is investigated at precise values of one variable and average values of another.

In case where the $\nu$-dependence is studied,

$$
R_{M}^{h}(\nu,\langle z\rangle)=2 d \sigma_{A}(\nu,\langle z\rangle) / A d \sigma_{D}(\nu,\langle z\rangle)
$$

where $\langle z\rangle$ are the average values of $z$ for each $\nu$ bin. And for $z$ - dependence

$$
R_{M}^{h}(\langle\nu\rangle, z)=2 d \sigma_{A}(\langle\nu\rangle, z) / A d \sigma_{D}(\langle\nu\rangle, z),
$$

where $\langle\nu\rangle$ are the average values of $\nu$ for each $z$ bin.

In this work we adopt a model, according to which the origin of NA is the absorption of the prehadron (string, dipole) and final hadron in the nuclear medium. In that case NA ratio has the following form:

$$
R_{M}^{h}=\int d^{2} b \int_{-\infty}^{\infty} \rho(b, x)[W(b, x)]^{(A-1)} d x,
$$

\footnotetext{
${ }^{1}$ In fact, $R_{M}^{h}$ also depends on the photon virtuality $Q^{2}$ and on the square of the hadron transverse momentum $p_{t}^{2}$, in respect to the virtual photon direction. However, from the experimental data, it is known that $R_{M}^{h}$ is a much sensitive function of $\nu$ and $z$ in comparison with $Q^{2}$ and $p_{t}^{2}$.
} 
where $W(b, x)$ is the probability that neither the prehadron produced at the DIS point $(b, x)$ (where $b$ is impact parameter and $x$ the longitudinal coordinate), nor the hadron produced at point $\left(b, x^{\prime}\right)$ is absorbed by one nucleon in the nucleus; $\rho$ is the nuclear density function with a normalization condition:

$$
\int \rho(r) d^{3} r=1
$$

For $W(b, x)$ we use the one scale model proposed in Ref. [5]:

$$
\begin{array}{r}
W(b, x)=1-\sigma_{q} \int_{x}^{\infty} P_{q}\left(x^{\prime}-x\right) \rho\left(b, x^{\prime}\right) d x^{\prime}- \\
\sigma_{h} \int_{x}^{\infty} P_{h}\left(x^{\prime}-x\right) \rho\left(b, x^{\prime}\right) d x^{\prime},
\end{array}
$$

where $\sigma_{q}$ and $\sigma_{h}$ are the inelastic cross sections for prehadron-nucleon and hadron-nucleon interactions, respectively. $P_{q}\left(x^{\prime}-x\right)$ is the probability that on distance $x^{\prime}-x$ from DIS point particle is a prehadron and $P_{h}\left(x^{\prime}-x\right)$ is the probability that particle is a hadron.

$$
P_{h}\left(x^{\prime}-x\right)=1-P_{q}\left(x^{\prime}-x\right) .
$$

In analogy with survival probability for a particle having lifetime $\tau$ in a system where it travels a distance $x^{\prime}-x$ before decaying, $P_{q}\left(x^{\prime}-x\right)$ can be expressed in form:

$$
P_{q}\left(x^{\prime}-x\right)=\exp \left[-\left(x^{\prime}-x\right) / \tau\right],
$$

where $\tau$ is the formation time. Substituting expressions for $P_{q}\left(x^{\prime}-x\right)$ and $P_{h}\left(x^{\prime}-x\right)$ in eq.(6) we obtain:

$$
\begin{array}{r}
W(b, x) \approx 1-\sigma_{h} \int_{x}^{\infty} \rho\left(b, x^{\prime}\right) d x^{\prime}+\tau\left(\sigma_{h}-\sigma_{q}\right) \rho(b, x) \\
\approx w_{1}(b, x)+\tau(\nu, z) w_{2}(b, x) .
\end{array}
$$

$W$ depends on $\nu$ and $z$ only by means of $\tau(\nu, z)$.

In more detail, the formation time in string models can be divided in two parts (see, for instance, two scale model presented in Ref [2, 6]). First past is the constituent formation time $\tau_{c}$, which defines the time elapsed from the moment of the DIS untill the production of the first constituent of the final hadron. Second time interval begins with the production of the first constituent until the second one, which coincides with the yo-yo ${ }^{2}$ or final hadron production. Comparison with the experimental data shows that in the second interval, the prehadron-nucleon cross section has values close to the

\footnotetext{
2 The yo-yo formation means that a colorless system with valence contents and quantum numbers of the final hadron is formed, but without its "sea" partons.
}

hadron-nucleon cross section $\sigma_{h}$. If the difference between these cross-sections is neglected, the model is reduced to one scale model with $\tau=\tau_{c}$. In case of the improved two scale model Ref [6], the prehadron-nucleon cross section reaches hadron-nucleon cross section value during a time interval $\tau=\tau_{c}+c \Delta \tau$, where $\Delta \tau=z \nu / \kappa$, $\kappa$ is the string tension, $\mathrm{c}$ is the free parameter which defines from fit. In Ref [6] it is shown that $c \ll 1$. Transition to the one scale model takes place at $\mathrm{c}=0$ and corresponds to $\tau=\tau_{c}$. One should note that any complicated absorption string model, in some approximation, can be reduced to the one scale model presented in eq.(6) and (9). Substituting $W(b, x)$ in $R_{M}^{h}$ we obtain:

$$
\begin{aligned}
& R_{M}^{h} \approx \int d^{2} b \int_{-\infty}^{\infty} \rho(b, x)\left(w_{1}+\tau w_{2}\right)^{(A-1)} d x \\
& \approx a_{i 1}+\tau a_{i 2}+\tau^{2} a_{i 3}+\cdots
\end{aligned}
$$

where $i$ is the maximal power of $\tau$ with which we are limited. The coefficients $a_{i j}$ depend on $A, \sigma_{q}, \sigma_{h}$ and nuclear density. This means, that $a_{i j}$ vary for different nuclei. For each nucleus $a_{i j}$ are the same for hadrons with equal values of $\sigma_{q}$ and $\sigma_{h}$ (for instance for pions ${ }^{3}$ and negatively charged kaons). Although $R_{M}^{h}$ is a polynomial of $\tau$ with maximal power $A-1$, it is expected that $a_{i 1}>a_{i 2}>a_{i 3}>\cdots$. For fitting of the NA data we use three expressions for $R_{M}^{h}$ as first, second and third order polynomials of $\tau$ :

$$
\begin{gathered}
P_{1}=a_{11}+\tau a_{12}, \\
P_{2}=a_{21}+\tau a_{22}+\tau^{2} a_{23}, \\
P_{3}=a_{31}+\tau a_{32}+\tau^{2} a_{33}+\tau^{3} a_{34} .
\end{gathered}
$$

In order to get the information on the influence of highest order of polynomials, also $P_{4}$ polynomial was checked (see section 4).

\section{FORMATION TIME}

Equation (10) shows, that within our approximation, $R_{M}^{h}$ is a function of $\tau$ only. In this section we will discuss the physical meaning and possible expression of the formation time $\tau$. There are different definitions for the formation time. We define formation time as a time scale within which the prehadron-nucleon cross section reaches the value of the hadron-nucleon one. In the literature there are three qualitatively different definitions for $\tau$. In the first extreme case it is assumed that $\tau=0$

\footnotetext{
3 we do not mention the electric charge of pions, because cross sections of differently charged pions with nucleons, which are of interest to us, are equal.
} 
(Glauber approach). In the second extreme case $\tau \gg r_{A}$, where $r_{A}$ is the nuclear radius (energy loss model [7]). And at last, in our opinion more realistic definition of the formation time, as a function of $\nu$ and $z$ which can change from zero up to values larger than $r_{A}$. Experimental data seem to confirm that for moderate values of $\nu$ on the order of $10 \mathrm{GeV}$ the formation time is smaller than the nuclear size, i.e. the hadronization takes place within the nucleus. We will use the formation times according to the third definition mentioned above. Following expressions are used:

1. Formation time for the leading hadron [8], which follows from the energy-momentum conservation law

$$
\tau_{\text {lead }}=(1-z) \nu / \kappa,
$$

where $\kappa$ is the string tension (string constant) with numerical value $\kappa=1 \mathrm{GeV} / \mathrm{fm}$. Indeed, eq. (14) presents formation time for the hadron produced on the fast end of the string or, which is the same, for the last hadron produced from string. Hadrons can be produced along whole length of the string. Among them, the hadrons produced on the fast end have better chance to avoid interactions in the nucleus.

2. Formation time for the fast hadron, which is composed of characteristic formation time of the hadron $h$ in its rest frame $\tau_{0}$ and Lorentz factor (see Ref. [5])

$$
\tau_{\text {Lor }}=\tau_{0} \frac{E_{h}}{m_{h}}=\tau_{0} \frac{z \nu}{m_{h}},
$$

where $E_{h}$ and $m_{h}$ are the energy and mass of the hadron $h$, respectively. Let us briefly discuss the factor $\tau_{0}$. Some authors assume (see Ref. [9]) that $\tau_{0}$ is a universal quantity which does not depends on the hadron type. If this assumtion is correct, then $\tau_{L o r}$ for the kaons is approximately 3.5 times shorter than for the pions at the same values of $\nu$ and $z$, although the experiment gives $R_{M}^{\pi} \approx R_{M}^{K^{-}}$, and it is known that $\sigma_{\pi} \approx \sigma_{K^{-}}$. Such definition seems merely strange in framework of the absorption model. It appears more realistic that $\tau_{0}$ is proportional to $m_{h}$. The reason for this is that in the string model the meson (baryon) is represented as a system consisting of a quark-antiquark (diquark) and a gluonic string between them. The energy of the system is transfered by the gluonic string from one parton to another and back. One full cycle lasts a period of $m_{h} / \kappa$ which we adopt as $\tau_{0}$. Then $\tau_{L o r}$ is a universal quantity which does not depend on the hadron type.

3. Formation time following from the Lund string model Ref. [10] ${ }^{4}$

$$
\tau_{\text {Lund }}=\left[\frac{\ln \left(1 / z^{2}\right)-1+z^{2}}{1-z^{2}}\right] \frac{z \nu}{\kappa} .
$$

\footnotetext{
${ }^{4}$ Note that this approximation is used only for the sake of easy reading. For numerical calculations we use the precise expression for $\tau_{\text {Lund }}$ following from equation $\tau_{\text {Lund }}=\tau_{y}-z \nu / \kappa$ with $\tau_{y}$ taken from eq.(4.21) of Ref. [10].
}

One should note that all three types of formation time have similar behaviour with $\nu$, but different behaviour with $z$. At the values of $z$ typical for the HERMES kinematics the behavior of $\tau$ defined as (14) and (16) is similar, i.e. they are decreasing with the increase of $z$, while $\tau$ defined as (15) is increasing with the increase of $z$.

\section{RESULTS}

A combined fit was performed for each nucleus and all hadrons having equal cross sections and, consequently, identically absorbed by nuclear matter (the same applies to their prehadrons). It is worth reminding, that the inelastic cross sections of corresponding hadrons with nucleons in the moderate energy range $\left(E_{h} \sim 10 \mathrm{GeV}\right)$ have the following values: $\sigma_{\pi}=\sigma_{K^{-}}=$ $20 \mathrm{mb}, \sigma_{K^{+}}=14 \mathrm{mb}, \sigma_{p}=32 \mathrm{mb}$ and $\sigma_{\bar{p}}=42 \mathrm{mb}$. Combined fit was performed only for the hadrons which have equal cross-sections. As a result, two combined

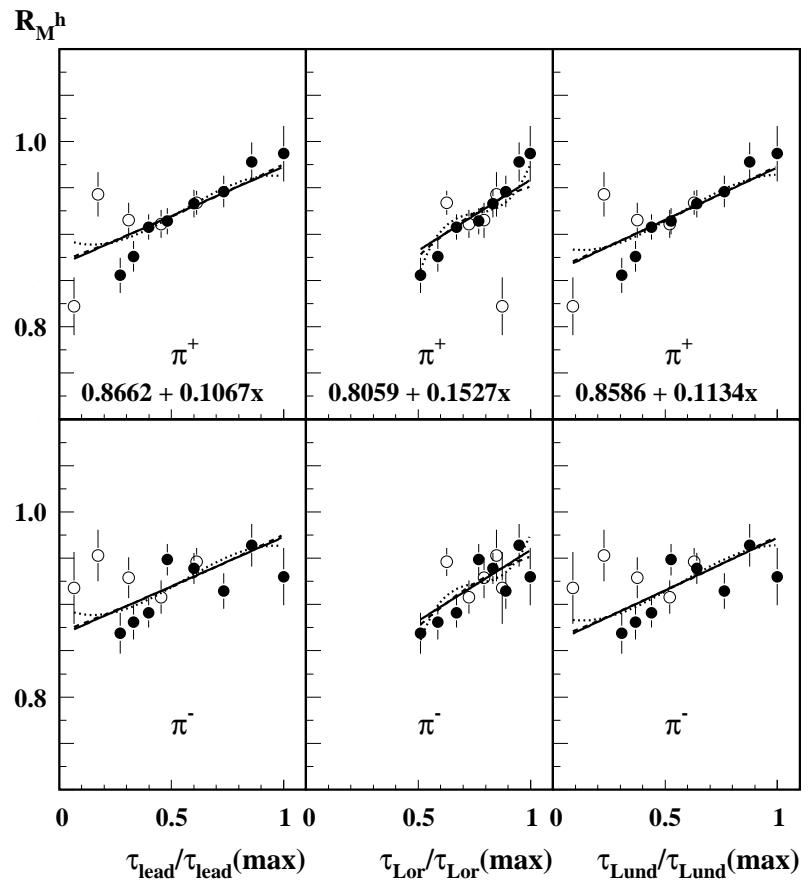

FIG. 1: The values $R_{M}^{h}$ on nitrogen as a function of $\tau_{\text {lead }}$ (left two panels), $\tau_{\text {Lor. }}$ (central two panels), $\tau_{\text {Lund }}$ (right two panels). Normalized values $\tau / \tau(\max )$ for all $\tau$ are used. On upper panels $\pi^{+}$, on lower $\pi^{-}$mesons are presented respectively. Solid, dashed and dotted curves are results of linear, quadratic and cubic polynomial fits. The numerical results for linear fit are noted on upper panels.

fits were performed for:

1. positive and negative pions on nitrogen (26 experimental points from [3]);

2. positive, negative, neutral pions and negative kaons on krypton (63 experimental points from [4]); 


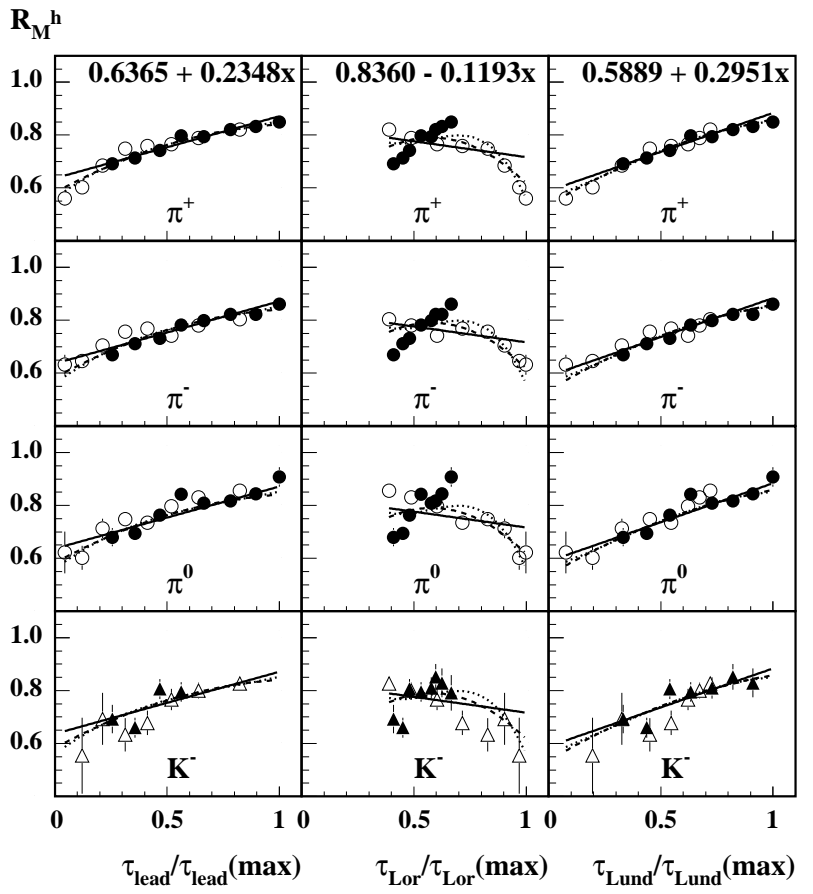

FIG. 2: The values $R_{M}^{h}$ on krypton as a function of $\tau_{\text {lead }}$ (left four panels), $\tau_{\text {Lor. }}$ (central four panels), $\tau_{\text {Lund }}$ (right four panels). On panels from upper to lower $\pi^{+}, \pi^{-}, \pi^{0}$ and $K^{-}$mesons, are presented respectively.

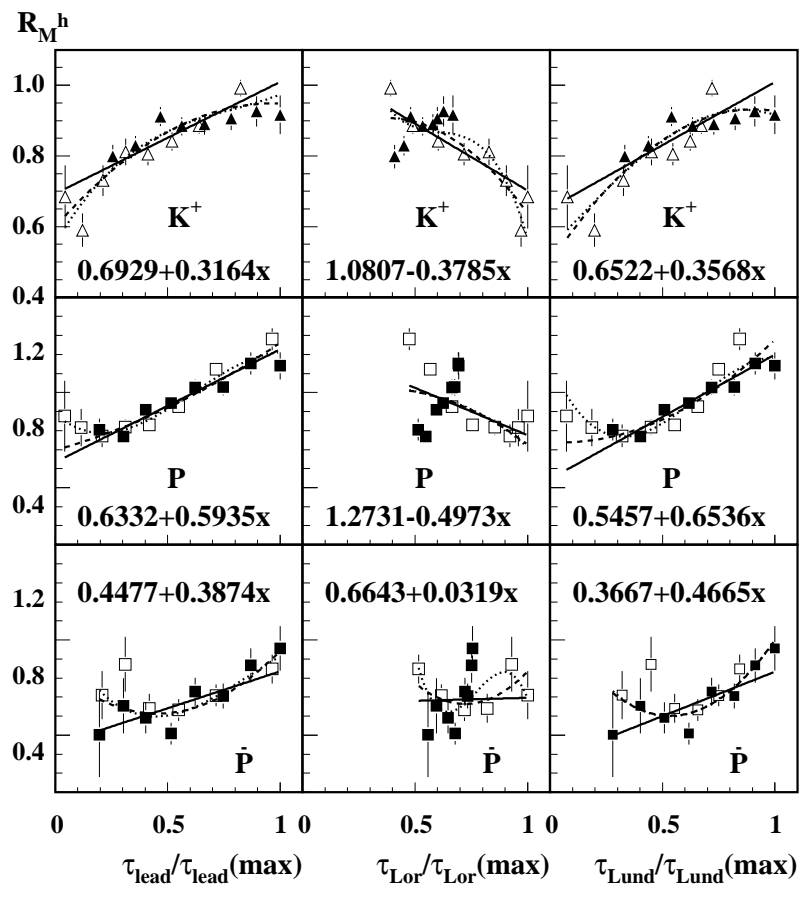

FIG. 3: The values $R_{M}^{h}$ on krypton as a function of $\tau_{\text {lead }}$ (left three panels), $\tau_{\text {Lor. }}$ (central three panels), $\tau_{\text {Lund }}$ (right three panels). The results for $K^{+}$mesons are presented on upper panels, protons - on middle, and antiprotons - on lower.
3. and separate fits for:

- positive kaons on krypton (16 experimental points [4]);

- protons on krypton (16 experimental points [4]) and

- antiprotons on krypton (14 experimental points [4]).

The NA ratios were taken in polynomial form (11)-(13), and formation times (lengths) as in (14)-(16). The results for the reduced $\chi^{2}$ denoted as $\chi^{2} /$ d.o.f. are presented in Table I. One can see that for each choice of formation time and for each nucleus, the values of $\chi^{2} /$ d.o.f. are close for the polynomials $P_{1}, P_{2}$ and $P_{3}$, which means that including higher power polynomials of $\tau$ will not essentially improve the description of data. In order to test this, we calculated also $P_{4}$ polynomials and obtained the values of $\chi^{2} /$ d.o.f. close to the ones in case of $P_{3}$. From Table Ione can see that the fit gives unexpectedly good values for $\chi^{2} /$ d.o.f. close to unity for $\tau_{\text {lead }}$ and $\tau_{\text {Lund }}$, and for $\tau_{\text {Lor }}$ the agreement is much worse. Experimental points as a function of $\tau$ and results of the fit are presented in Fig. 1 for nitrogen and in Figures 2,3 for krypton. Solid points correspond to the NA ratio obtained from the experimental data for $\nu$-dependence, open points for $z$-dependence. For convenience we have renormalized $\tau$ to $\tau / \tau(\max )$, where $\tau(\max )$ are the $\max -$ imum values of $\tau$ for each set of data and each choice of $\tau$ expression. On each of the figures the linear polynomial is presented $a_{11}+\tau a_{12}^{\prime}$ with values $a_{11}$ and $a_{12}^{\prime}=$ $a_{12} \tau(\max )$ corresponding to the best fit. Solid, dashed and dotted curves correspond to $P_{1}, P_{2}$ and $P_{3}$ polynomial fit.

\section{CONCLUSIONS.}

- Based on the performed studies one can assume that NA ratio is a function of formation time $\tau$ only.

- Three expressions for NA ratio as a first, second and third order polynomials of $\tau$ were used for these studies, and the results do not show remarkable difference in their ability to describe the HERMES data.

- The performed analysis with three different expressions for the formation time $\tau$ shows that based on the obtained values of the reduced $\chi^{2}$ (see Table 1), as well as on visual shape of the curves in Figures 1, 2] and 3, one can conclude that the two of them $-\tau_{\text {lead }}$ and $\tau_{\text {Lund }}$ are quite appropriate, and the expression for the formation time in form of $\tau_{\text {Lor }}$ is ruled out.

- The performed combined fits are based on the HERMES NA data using: $\pi^{+}, \pi^{-}$for nitrogen; $\pi^{+}$, 
$\pi^{-}, \pi^{0}, K^{-}$for krypton; separate fits for $K^{+}$, protons and antiprotons for krypton.

- Because of the fact that the linear function gives about the same values $\chi^{2} /$ d.o.f. values as second and third order one, we assume that in our approach the parameterization by linear function is well enough.
[1] L.S. Osborn et al., Phys. Lett. 40B, (1978), 1624

[2] J.Ashman et al., Z.Phys. C52(1991) 1

[3] A.Airapetian et al., Eur.Phys. J. C20 (2001) 479

[4] A.Airapetian et al., Phys.Lett. B577 (2003) 37

[5] A.Bialas, Acta Phys.Pol. B11 (1980) 475
[6] N.Akopov, L.Grigoryan, Z.Akopov, Eur.Phys.J. C44 (2005) 219; hep-ph/0409359 (2004)

[7] X.-N. Wang, X. Guo, Nucl. Phys., A696 (2001), 788; E. Wang, X.-N. Wang, Phys. Rev. Lett. 89 (2002), 162301

[8] B.Kopeliovich, Phys.Lett. B243 (1990) 141

[9] T.Falter et al., Phys.Lett. B594 (2004) 61

[10] A.Bialas, M.Gyulassy, Nucl.Phys. B291 (1987) 793 
TABLE I: $\chi^{2} /$ d.o.f. from polynomial fit. Details see in text

\begin{tabular}{|c|c|c|c|c|c|c|c|c|c|c|c|}
\hline \multicolumn{2}{|c|}{} & \multicolumn{3}{|c|}{$\tau_{\text {lead. }}$} & \multicolumn{3}{|c|}{$\tau_{\text {Lor }}$} & \multicolumn{3}{|c|}{$\tau_{\text {Lund }}$} \\
\hline $\mathrm{A}$ & $N_{\text {exp }}$ & Had. & $P_{1}$ & $P_{2}$ & $P_{3}$ & $P_{1}$ & $P_{2}$ & $P_{3}$ & $P_{1}$ & $P_{2}$ & $P_{3}$ \\
\hline${ }^{14} N$ & 26 & $\pi^{+,-}$ & 1.65 & 1.72 & 1.73 & 1.89 & 1.95 & 1.81 & 1.60 & 1.67 & 1.71 \\
\hline${ }^{84} K r$ & 63 & $\pi^{+,-, 0}, K^{-}$ & 1.67 & 1.32 & 1.31 & 8.71 & 6.62 & 5.98 & 1.41 & 1.23 & 1.23 \\
\hline${ }^{84} K r$ & 16 & $K^{+}$ & 1.95 & 1.62 & 1.70 & 3.47 & 3.30 & 3.14 & 2.78 & 2.34 & 2.52 \\
\hline${ }^{84} K r$ & 16 & proton & 1.25 & 1.24 & 1.04 & 8.50 & 9.08 & 9.82 & 2.48 & 2.28 & 1.90 \\
\hline${ }^{84} K r$ & 14 & antipr. & 1.33 & 0.89 & 0.92 & 2.43 & 2.37 & 2.04 & 1.50 & 0.94 & 1.03 \\
\hline
\end{tabular}

